\title{
Satisfação, sobrecarga e opinião sobre doença mental de profissionais de saúde
}

Fernanda Pâmela Machado ${ }^{1}$

(D) https://orcid.org/0000-0002-2446-1341

Marcos Hirata Soares ${ }^{1}$

(D) https://orcid.org/0000-0002-1391-9978

Layla Karina Ferrari Ramos ${ }^{1}$

(D) https://orcid.org/0000-0002-4306-2207

Eloisi Delalibera Ruzzon 1

(D) https://orcid.org/0000-0002-2523-9515
Universidade Estadual de Londrina, Departamento de Enfermagem, Londrina, PR, Brasil.
Objetivo: avaliar profissionais da rede de cuidados em saúde mental através de sua atitude, satisfação e sobrecarga. Método: estudo transversal, com 46 profissionais das equipes da Rede de Atenção Psicossocial de uma cidade no Norte do Paraná. O questionário foi dividido em 4 partes: Caracterização sócio ocupacional; Escala de Avaliação da Satisfação da Equipe em Serviços de Saúde Mental; Escala de Avaliação do Impacto do Trabalho em Serviços de Saúde Mental; Escala de Opiniões sobre a Doença Mentat. Resultados: 65,8\% dos profissionais, deram-se por satisfeitos com a rede de saúde mental. Em relação às condições físicas e o conforto do serviço, 53,4\% não se encontraram satisfeitos, expressando possibilidade de melhora. Conclusão: a avaliação dos serviços de saúde mental é relevante quando se almeja melhorias na qualidade dos serviços ofertados.

Descritores: Saúde Mental; Satisfação Pessoal; Carga de Trabalho; Serviços de Saúde Mental.

\section{Como citar este artigo}

Machado FP, Soares MH, Ramos LKF, Ruzzon ED. Satisfaction, overload and opinion of health professionals on mental disease. SMAD, Rev Eletrônica Saúde Mental Álcool Drog. 2021 abr.-jun.;17(2):26-33. doi: https://dx.doi.org/10.11606/issn.1806-6976.smad.2021.152995 


\section{Satisfaction, overloading and opinion on mental disease of health professionals}

Objective: to evaluate professionals in the mental health care network through their satisfaction and overload. Method: cross-sectional study with 46 professionals from the teams of the Psychosocial Care Network in a city in Northern Paraná. The questionnaire was divided into 4 parts: Sociooccupational characterization; Rating Scale for Team Satisfaction in Mental Health Services; Scale of Assessment of the Impact of Work on Mental Health Services; Scale of Opinions on Mental Illness.

Results: $65.8 \%$ of the professionals were satisfied with the mental health network. Regarding the physical conditions and comfort of the service, $53.4 \%$ are not considered, expressing the possibility of improvement. Conclusion: the assessment of mental health services is relevant when aiming for improvements in the quality of the services offered.

Descriptors: Mental Health; Personal Satisfaction; Workload; Mental Health Services.

\section{Satisfacción, sobrecarga y opinión de los profesionales de la salud sobre enfermedad mental}

Objetivo: evaluar a los profesionales de la red de salud mental a través de su actitud, satisfacción y sobrecarga. Método: estudio transversal con 46 profesionales de los equipos de la Red de Atención Psicosocial en una ciudad del norte de Paraná. El cuestionario se dividió en 4 partes: Caracterización sociolaboral; Escala de calificación para la satisfacción del equipo en los servicios de salud mental; Escala de evaluación del impacto del trabajo en los servicios de salud mental; Escala de opiniones sobre enfermedades mentales. Resultados: el 65,8\% de los profesionales se mostró satisfecho con la red de salud mental. En cuanto a las condiciones físicas y la comodidad del servicio, el 53,4\% no se mostró satisfecho y expresó la posibilidad de mejora. Conclusión: la valoración de los servicios de salud mental es relevante a la hora de buscar mejoras en la calidad de los servicios ofrecidos.

Descriptores: Salud Mental; Satisfacción Personal; Carga de Trabajo; Servicios de Salud Mental. 


\section{Introdução}

Desde os primórdios da Reforma Psiquiátrica, debate-se a atenção adequada à saúde mental. O profissional de saúde é capacitado para poder atuar em diversas áreas e conviver com pacientes que apresentem diferentes diagnósticos, do menos complexo ao mais complexo, dentre eles, o portador de doença mental. A doença mental ainda é muito estigmatizada, e os próprios profissionais de saúde o fazem, tornando-se uma barreira para o paciente e, também, para os profissionais, ocasionando insatisfação e sobrecarga no ambiente de trabalho(1-2).

Estudos apontam a relevância em realizar avaliações, visando melhorar a qualidade dos serviços em saúde mental. Essas avaliações, envolvem os usuários do serviço, a participação de seus familiares e os profissionais que ali atuam, proporcionando um elo maior entre os mesmos. Uma avaliação integral é estimulada com o objetivo de estimar o nível de satisfação dos três segmentos e o grau de sobrecarga de familiares e profissionais dos serviços ${ }^{(3)}$.

Estudo realizado no Brasil constatou um alto grau de satisfação dos usuários do Centro de Atenção Psicossocial (CAPS), onde o principal implicador da satisfação são as más condições de infraestrutura, impactando negativamente na insatisfação de profissionais de saúde que trabalham com a saúde mental(3-4).

Alguns estudos ${ }^{(5-6)}$ sugerem que os CAPS devem ser submetidos a processos avaliativos, de modo a verificar a qualidade de atenção à saúde ofertada nessas instituições, além das avaliações dos serviços por estarem atrelados ao planejamento e gestão dos serviços de saúde. Um efetivo sistema de avaliação deve reordenar a execução das ações e serviços, redimensionando-os de forma a alcançar as necessidades de seus usuários, dando maior racionalidade ao uso dos recursos.

A percepção dos pacientes, familiares e dos profissionais são perspectivas diferentes, quando se concebe o processo de avaliação de um serviço de saúde, mas também, são complementares, pois propõe uma avaliação integral de um serviço de saúde ${ }^{(7)}$.

Uma pesquisa com profissionais envolvidos no processo de cuidado em saúde mental( ${ }^{(8)}$ destaca que os perfis atitudinais mais positivos estão diretamente ligados à vivência do prazer no trabalho. Sugeriu-se que fosse ofertado a esses profissionais um programa de aprimoramento profissional para que o aprofundamento do conhecimento sobre a doença mental e mudança de seus perfis atitudinais possam ser mais condizentes com as propostas dos serviços substitutivos em saúde mental.

No entanto, há necessidade de se avaliar profissionais da rede de cuidados em saúde mental quanto às suas atitudes, satisfação e a sobrecarga, sendo estas variáveis importantes dentro do processo avaliativo, por refletirem outros aspectos importantes da qualidade, mas também, das boas condições de trabalho.

\section{Método}

Trata-se de um estudo descritivo, transversal, realizado no Centro de Atenção Psicossocial (CAPS) III Centro de Atenção Psicossocial Infantil (CAPS i) e Núcleo de Apoio à Saúde da Família (NASF) no ano de 2016.

A população do estudo foi composta por profissionais de nível superior e médio (assistentes sociais, enfermeiros, médicos, psicólogos, terapeutas ocupacionais, técnicos de enfermagem, artesão e outros). Para estes, o critério de inclusão foi de atuar como profissionais nos serviços da rede de cuidados em saúde mental por no mínimo 3 meses.

A pesquisa foi aprovada pelo comitê de Ética em Pesquisa com Seres Humanos (CEP), sob a CAAE n. 18426114.7.0000.5231. Os profissionais foram procurados pessoalmente, e entrevistados em seus horários disponíveis, após autorização do CEP. A amostra foi composta por 46 profissionais dos serviços, que atuam em período matutino ou vespertino. Para os participantes, garantiu-se o anonimato, o sigilo das informações e o direito de recusa ou abandono da pesquisa em qualquer etapa. Estas garantias foram asseguradas no Termo de Consentimento Livre e Esclarecido que foi assinado após a explicação dos objetivos da pesquisa e esclarecimento de dúvidas.

\section{Coleta de dados}

Foi aplicado um questionário contendo 4 partes: 1 ) Caracterização sócio ocupacional; 2) Escala de Avaliação da Satisfação da Equipe em Serviços de Saúde Mental (SATIS-BR/equipe); 3) Escala de Avaliação do Impacto do Trabalho em Serviços de Saúde Mental (IMPACTO-BR); 4) Escala de opiniões sobre a doença mental (ODM).

A SATIS-BR versão abreviada contém 32 itens quantitativos, cada um com respostas dispostas em escala Likert de cinco pontos, correspondendo a: 1"muito insatisfeito", 2- "insatisfeito", 3- "indiferente", 4 - "satisfeito", 5- "muito satisfeito". Estes itens são agrupados em 4 sub-escalas ou fatores: a) satisfação em relação à qualidade dos serviços oferecidos (fator 1 ); b) satisfação da equipe com relação à sua participação no serviço (fator 2 ); c) satisfação da equipe em relação às condições de trabalho (fator 3 ); satisfação da equipe a respeito do seu relacionamento no serviço (fator 4 ). Além disso, 3 questões qualitativas foram incluídas no final do questionário.

A escala de IMPACTO-BR contém 18 itens, cada um com respostas dispostas em escala Likert de cinco pontos que correspondem a: 1- "de forma alguma", 2- "não muito", 3- "mais ou menos", 4- "muito", 5"extremamente". Os itens são agrupados em três fatores: efeitos sobre a saúde física e mental; efeitos no funcionamento da equipe; repercussões emocionais do trabalho. 
A ODM foi traduzida e validada para o Brasil $^{(6)}$ e é composta de 51 afirmações. Suas opções de respostas variam segundo uma sequência progressiva de seis pontos de concordância, desde "concordo totalmente" até "discordo totalmente". Essas afirmações estão agrupadas em sete fatores: autoritarismo, benevolência, ideologia de higiene mental, restrição social, etiologia interpessoal, etiologia de esforço mental e visão minoritária.

Foram feitas análises estatísticas descritivas exploratória e testes de correlação entre os escores dos instrumentos aplicados. Os dados foram analisados no Programa Statistical Package for the Social Sciences, v.21. O nível de significância adotado foi de $a=0.05$.

\section{Resultados}

Da população estudada, 38 entrevistados eram do sexo feminino e 7 do sexo masculino. A média de idade foi de 41,95 anos, com variação entre 28 e 63 anos. A Tabela 1 apresenta os principais dados sócio-demográficos da população estudada.

Tabela 1 - Categoria de profissionais, número e porcentagem do grau de escolaridade e atuação em serviço de saúde mental. Paraná, Brasil, 2016

\begin{tabular}{lcc}
\hline Grau de escolaridade/atuação & $\mathbf{N}^{\circ}$ & $\%$ \\
\hline Nível médio & 6 & $13,3 \%$ \\
Nível superior & 12 & $26,7 \%$ \\
Pós-graduação & 27 & $60,0 \%$ \\
CAPS III & 21 & $46,7 \%$ \\
CAPS I & & $24,4 \%$ \\
NASFł & 11 & $28,9 \%$ \\
Enfermeiro & 13 & $15,6 \%$ \\
Médico & 7 & $8,9 \%$ \\
Psicólogo & 4 & $28,9 \%$ \\
Assistente Social & 13 & $6,7 \%$ \\
Terapeuta Ocupacional & 3 & $4,4 \%$ \\
Auxiliares/técnicos de enfermagem & 2 & $13,3 \%$ \\
Artesão & 6 & $2,2 \%$ \\
Outras profissões & 1 & $20,0 \%$ \\
\hline
\end{tabular}

${ }^{*}$ CAPS III = Centro de Atenção Psicossocial III; ${ }^{+}$CAPS I = Centro de Atenção Psicossocial Infantil; ${ }^{\ddagger}$ NASF $=$ Núcleo de Apoio à Saúde da Família

Quanto à formação acadêmica $60 \%$ possuem pósgraduação e 13,3\% possuem nível médio. Quanto ao tipo de serviço, 46,7\% atuam em CAPS III, 28,9\% em NASF e $24,4 \%$ em CAPS. A Tabela 2 relaciona os escores de média e desvio padrão das escalas SATIS-BR, Impacto e Opinião sobre a doença mental, em profissionais que atuam na assistência a pessoas com transtornos mentais. 1- "muito insatisfeito", 2- "insatisfeito", 3- "indiferente", 4 - "satisfeito", 5- "muito satisfeito.
Tabela 2 - Escores de média $(\mathrm{m})$ e desvio padrão ( $\mathrm{dp}$ ) da escala SATIS-BR* ${ }^{*}$ Impacto e Opinião sobre doença mental, em profissionais de $\mathrm{CAPS}^{\dagger}$ e NASF${ }^{\ddagger}$. Paraná, Brasil, 2016

\begin{tabular}{lcc}
\hline SATIS-BR* & $\mathbf{m}^{\S}$ & dp\| \\
\hline $\begin{array}{l}\text { Satisfação no trabalho } \\
\text { Qualidade dos serviços oferecidos aos }\end{array}$ & 3,29 & 0,65 \\
pacientes (S-1) & 3,36 & 0,56 \\
Participação da equipe no serviço (S-2) & 3,30 & 0,72 \\
Condições de trabalho (S-3) & 2,92 & 0,61 \\
Relacionamento no serviço (S-4) & 3,58 & 0,73 \\
\hline Impacto da carga de trabalho & $\mathbf{m}$ & $\mathbf{d p}$ \\
Impacto & 1,83 & 0,59 \\
Saúde física e mental (I-1) & 1,67 & 0,65 \\
Sobrecarga no trabalho (I-2) & 1,76 & 0,51 \\
Efeitos no estado emocional (I-3) & 2,05 & 0,61 \\
\hline Opiniões sobre doença mental & $\mathbf{m}$ & $\mathbf{d p}$ \\
Autoritarismo (A) & 4,90 & 1,87 \\
Benevolência (B) & 5,02 & 1,19 \\
Ideologia de higiene mental (C) & 4,04 & 2,11 \\
Restrição social (D) & 5,38 & 1,79 \\
Etiologia interpessoal (E) & 3,22 & 1,27 \\
Etiologia de esforço mental (F) & 4,90 & 1,25 \\
Visão minoritária (G) & 3,95 & 1,4 \\
\hline
\end{tabular}

*SATIS-BR = Escala de Avaliação da Satisfação da Equipe em Serviços de Saúde Mental; ${ }^{+}$CAPS = Centro de Atenção Psicossocial; ${ }^{\ddagger}$ NASF = Núcleo de Assistência à Saúde da Família; ${ }^{\S} \mathrm{m}=$ Média; $\| \mathrm{dp}=$ Desvio Padrão

Em relação à satisfação dos profissionais de saúde obteve-se um escore global de 3,29, equivalendo a $65,8 \%$ do conceito máximo de satisfação possível, sugerindo, então, o grau de satisfação dos profissionais quanto à qualidade dos serviços ofertados aos pacientes (Tabela 2). Quando se observa o impacto da carga de trabalho, obtém-se um escore global de 1,83, equivalendo a $37,8 \%$ do conceito máximo esperado. Ou seja, o impacto global foi menor, acompanhando o escore mais elevado de satisfação no trabalho.

Quanto à opinião sobre a doença mental, os domínios Benevolência obteve um escore de 5,02 condizendo que o profissional desta área traduz que ocorre que o doente mental devido à infelicidade do indivíduo, assim devendo estes profissionais ampará-los por meio protecionista e bondoso.

Já o escore de Restrição social, 5,38, representa e traduz a visão destes trabalhadores, no qual a doença mental é um defeito hereditário, no qual o portador pode contaminar sua família e a sociedade, devendo ser protegida pelos direitos pessoais e sociais do paciente. Ao analisar o nível de significância estatística dos fatores da escala ODM, apenas os domínios Etiologia interpessoal e Visão minoritária não foram aprovados no Teste de Hipóteses ( $p>0.05$ ).

A Tabela 3 apresenta a correlação de Pearson ( $r)$, a variância compartilhada $\left(r^{2}\right)$ e o valor de $p(p)$ entre os domínios das escalas SATIS-BR e IMPACTO-BR. 
Tabela 3 - Coeficiente de correlação de Pearson $(r)$, variância compartilhada $\left(r^{2}\right)$ e valor- $p(p)$ entre as escalas SATIS-BR* ${ }^{*}$ e IMPACTO-BR ${ }^{\dagger}$. Paraná, Brasil, 2016

\begin{tabular}{|c|c|c|c|c|c|}
\hline & \multicolumn{5}{|c|}{ Satisfação No Trabalho } \\
\hline \multirow{10}{*}{ 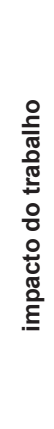 } & & $S-1^{\ddagger}$ & $\mathrm{S}-2^{\mathrm{s}}$ & S-3" & $S-4 \pi$ \\
\hline & $1-1^{* *}$ & $-0,32$ & $-0,30$ & $-0,35$ & $-0,37$ \\
\hline & $r^{t+1 / r^{2+f}}$ & $(10,24 \%)$ & $(9,24 \%)$ & $(12,25 \%)$ & $(13,54 \%)$ \\
\hline & $p^{\S \S}$ & 0,035 & 0,045 & 0,020 & 0,014 \\
\hline & I-2" & $-0,22$ & $-0,41$ & $-0,27$ & $-0,37$ \\
\hline & $r^{+t /} / r^{2 \neq}$ & $(4,7 \%)$ & $(17,10 \%)$ & $(7,2 \%)$ & $(14,06 \%)$ \\
\hline & $p^{\S \S}$ & 0,155 & 0,004 & 0,078 & 0,012 \\
\hline & $|-3||| \mid$ & $-0,5$ & $-0,52$ & $-0,44$ & $-0,49$ \\
\hline & $r^{+t} / r^{2 \neq \ddagger}$ & $(25 \%)$ & $(26,93 \%)$ & $(19,53 \%)$ & $(24,30 \%)$ \\
\hline & $p \S \S$ & 0,001 & 0,000 & 0,003 & 0,001 \\
\hline
\end{tabular}

*SATIS-BR = Escala de Avaliação da Satisfação da Equipe em Serviços de Saúde Mental; ${ }^{+}$IMPACTO-BR = Escala de Avaliação do Impacto do Trabalho em Serviços de Saúde Mental; ${ }^{\ddagger}$ S1 = Qualidade dos Serviços Oferecidos aos Pacientes, ${ }^{5}$ S2 = Participação da Equipe no Serviço; "S3 = Condições de Trabalho; "S-4 = Relacionamento no Serviço; ${ }^{* *} \mathrm{I}-2$ e I2 = Saúde Física e Mental; ${ }^{++} r=$ Coeficiente de Correlação de Pearson, ${ }^{* \neq} r 2$ = Variância Compartilhada; ${ }^{{ }^{\S}} \mathrm{p}=$ Valor de $p$; $" \| \mathrm{I} 3=$ Efeitos no Estado Emocional

Conforme a Tabela 3, com exceção às correlações entre S-1 e I-2 e I-2 e S-3, que representam a qualidade no serviço oferecido aos pacientes e sobrecarga no trabalho e essa com condições de trabalho, respectivamente, todas as correlações foram estatisticamente significativas, denotando, então, a relação inversamente proporcional entre satisfação no trabalho e a sobrecarga com o mesmo.

\section{Discussão}

\section{Impacto e satisfação no trabalho}

Neste estudo, os profissionais se mostraram satisfeitos com sua participação nas tomadas de decisão no serviço, o que difere um pouco em outro estudo( ${ }^{(9)}$, onde os escores indicaram baixos índices de satisfação da equipe de enfermagem, e os escores geral de satisfação variaram de 2,2 a 4,8 e a média dos escores foram de 3,4 , os fatores que apresentaram maiores satisfação foi "relacionamentos", apresentando escore médio de 3,7, seguido pelos fatores "Qualidade do serviço e Condições de trabalho" com escores médios de 3,4.

Nesse contexto, outros estudos, destacam que o principal fator para profissional apresentar melhor satisfação no trabalho é necessário, no serviço de saúde mental, aprimorar a organização do trabalho e na infraestrutura dos serviços. Contudo, os maiores índices de satisfação se referem às condições de trabalho e ao relacionamento com os colegas no serviço. Esses resultados também foram encontrados em outros estudos, no qual a subescala com maior índice de satisfação foi a de relacionamento no serviço, com média 3,74 $\pm 0,57$ e a subescala que gerou menor nível de satisfação foi em relação às condições de trabalho com média 2,99 $\pm 0,56^{(10)}$.
Quanto aos fatores avaliados, observou-se maior sobrecarga no que se refere às repercussões emocionais do trabalho, sendo o trabalho em saúde mental não considerada sobrecarga emocional pelos profissionais. Esse resultado corrobora com outros dados obtidos(10-12) nos quais se constatou maior grau de sobrecarga nessa dimensão, contudo um menor impacto se referiu à saúde física e mental, confirmada também por dados apresentados em outros estudos ${ }^{(7,12-15)}$.

Observou-se correlação negativa significativa entre os escores de satisfação e sobrecarga, apresentando menores níveis de satisfação e maiores de sobrecarga, em profissionais com indicativo de estresse. Estes resultados confirmam a hipótese de que há uma associação entre estas variáveis, conforme esperado, atestando a validade convergente entre as escalas de satisfação (SATIS-BR) e de sobrecarga (IMPACTO-BR), aplicada aos profissionais de serviços de saúde mental.

Essas informações obtidas com estas escalas colaboram para avaliação dos serviços de saúde mental, sendo importantes indicadores de qualidade, uma vez que a satisfação e a sobrecarga sentidas pelos profissionais das equipes de trabalho, podem afetar diretamente a qualidade do atendimento prestado aos pacientes ${ }^{(13)}$.

Existe a necessidade na mudança de aspectos do funcionamento do serviço ou implantação de novos procedimentos de apoio e acompanhamento dos profissionais, para que sejam apoiados e incentivados aumentando seu nível de satisfação e amenizando a sobrecarga de trabalho, promovendo assim, impacto positivo, por diminuir a ocorrência de absenteísmo e possibilitar melhorias para profissionais e pacientes no seu ambiente laborativo(16).

Atitudes frente à Doença Mental

Os profissionais de serviço de urgência e emergência(17) de nível técnico e superior obtiveram uma mediana 10,0 com relação ao fator Sten no fator autoritarismo, no qual reflete a perspectiva de que o doente mental necessita ser isolado de outros pacientes, permanecendo sob portas trancadas e vigilância. Contém o conceito da irrecuperabilidade pessoal e social do doente e a ideia de sua periculosidade. A mediana para autoritarismo em nível técnico foi de 8,0 e para o nível superior de $6,0^{(13)}$.

O fator Benevolência sugere que o doente mental, devido à sua infelicidade, deve ser amparado por meio de protecionismo bondoso e paternalista, com base em cuidados, atenção pessoal e conforto material. Em profissionais de um serviço de urgência e emergência encontrou-se a mediana 1,0 para nível técnico e superior(17). Em profissionais de CAPS, centro de convivência e residências terapêuticas ${ }^{(18)}$ a mediana encontrada foi de 4,0 para os níveis técnico e superior. Comparando com de um estudo com enfermeiros de 
emergências em saúde mental, nota-se que os enfermeiros estudados são menos autoritários e mais benevolentes do que os enfermeiros de emergências psiquiátricas ${ }^{(17)}$ corroborando assim, com o estudo em questão.

Já o fator Ideologia da higiene mental(18) representa a ideia que o doente mental é uma pessoa semelhante às pessoas normais, com diferenças quantitativas, porém não qualitativas, podendo desempenhar atividades especializadas e até cuidar de crianças. Encontrou-se uma mediana de 5,0 para profissionais de nível técnico, atingindo a média, porém difere de outro estudo(18), onde profissionais de nível técnico apresentaram uma mediana 4,0 resultados abaixo da média, indicando ser menos semelhante as pessoas com doença mental.

Em relação à Restrição social(18), fator em que a doença mental é vista como uma espécie de defeito hereditário, completamente diferente de outras doenças, o portador pode contaminar a família e a sociedade, devendo, portanto, ser protegidas por meio de restrição dos direitos pessoais e sociais do paciente, mesmo após a hospitalização com uma mediana encontrada de 10,0, para profissionais de nível técnico indicando uma crença total de que esses pacientes apresentam transtornos hereditários, devendo permanecer no convívio de sua família. No entanto, um estudo(18) apresentou uma mediana de 7,0 apresentando-se acima da média 5,0.

O fator Etiologia interpessoal explica a doença mental como originária de vivências interpessoais, com ênfase para a interação com figuras parentais. Os sujeitos acreditam fortemente que os transtornos mentais são relacionados a fatores interpessoais ligados a graus de parentesco, com uma mediana de 8,0 correspondida por profissionais de nível técnico de um serviço de urgência e emergência, diferindo de outro estudo(18) onde os profissionais de nível técnico obtiveram a mediana de 5,0 equiparando a média.

O fator Etiologia de esforço mental reflete a ideia de que a doença mental se origina de "excessivo esforço cerebral" pelo trabalho intelectual exagerado, por pensar demais ou por ter pensamentos negativos, indicando que há uma crença total nestes profissionais de que essa doença é provinda de esforço mental exacerbado, sendo que, o estudo apresentou uma mediana de 10,0 para profissionais de nível técnico, essa mediana representa que esses profissionais acreditam que os transtornos mentais são hereditários e que estes pacientes devem permanecer em convívio familiar ${ }^{(17)}$.

O fator Visão minoritária representa o conceito de que o doente mental, por ser muito diferente das pessoas consideradas normais, pode ser facilmente reconhecido em um agrupamento humano, principalmente pela sua aparência externa, Segundo a tabela 2, foi encontrado o escore com uma média de 3,95; isso representa que o doente mental não é facilmente reconhecido em grupos de pessoas sem transtorno mental, sendo corroborado por um estudo ${ }^{(17)}$ com profissionais de saúde, serviços de emergência, os quais acreditam que estes pacientes são diferentes devido à sua aparência, apresentando uma média de 7,24.

O presente estudo corrobora com outros achados ${ }^{(18)}$, apesar de públicos diferentes, alguns voltados para alunos da graduação; pode-se considerar a grande lacuna de baixo conhecimento relacionado sobre a doença mental, porém, um estudo ${ }^{(19)}$ discorda deste levantamento, onde é apresentado em seus resultados que os alunos indicavam níveis de tolerância e sensibilidade sociais frente ao portador de doença mental.

\section{Conclusão}

A avaliação dos serviços de saúde permite verificar a qualidade dos serviços ofertados, sua efetividade e as falhas presentes, a fim de promover estratégias para melhoria. Os resultados também, em consonância com a literatura, apontam para a necessidade de maiores investimentos em recursos materiais e humanos. O que expõe a equipe a um trabalho estressante. Contudo, torna -se imprescindível uma infraestrutura mais adequada para o atendimento aos pacientes, visto que foi uma insatisfação grande por parte dos profissionais, em relação a poder realizar adequadamente as atividades com os pacientes.

Os resultados obtidos ainda apresentaram correlações significativas nas variáveis analisadas. Desse modo, pode-se concluir que as escalas utilizadas SATIS-BR, IMPACTO-BR e ODM foram instrumentos de medida apropriados para serem utilizados em estimativas do grau de satisfação, sobrecarga e estresse dos profissionais nos serviços de saúde mental. Essas escalas permitiram avaliar, separadamente pelas sub-escalas e com escores de medida independentes, diferentes dimensões dos serviços de saúde mental. As escalas de medida validadas padronizadas, são necessárias para avaliar esses serviços, fazendo com que haja confiabilidade e comparabilidade nos dados. Possibilitando assim a intervenção nos processos de trabalho, tornando-os o melhor possível.

Com base nesses resultados encontrados, sugere-se que sejam ofertados mais capacitações, aprimoramentos e educação continuada para os profissionais da área de saúde mental, visando uma melhoria da assistência a ser prestada e, que de fato, compreendam e saibam se relacionar com o portador de doença mental.

Além destes, pode-se enfatizar a importância dos profissionais buscarem por melhores infraestruturas, condições e organização no trabalho, experienciando assim, consequentemente, um bom relacionamento interpessoal entre os profissionais e, também com os pacientes portadores de doença mental. 


\section{Referências}

1. Cassiano APC, Marcolan JF, Silva DA da. Atenção primária à saúde: estigma a indivíduos com transtornos mentais. Rev Enferm UFPE. [Internet]. 2019;13(e):239668. [Acesso 20 abril 2020]; Disponível em: https://periodicos. ufpe.br/revistas/revistaenfermagem/article/view/239668. doi: https://doi.org/10.5205/1981-8963.2019.239668 2. Quinderé PHD, Bessa JMS, Batista FT. Rede de Atenção Psicossocial: qual o lugar da saúde mental?. Physis. [Internet]. 2014;24(1):253-71. [Acesso 18 abril 2020]; Disponível em: http://www.scielo.br/ scielo.php?script=sci_artext\&pid $=$ S0103733120140 00100253\&lng=en. doi: http://dx.doi.org/10.1590/ S0103-73312014000100014

3. Bandeira M, Silva MA da. Escala de Satisfação dos Pacientes com os Serviços de Saúde Mental (SATIS-BR): estudo de validação. J Bras Psiquiatr. [Internet]. 2012;61(3):124-32. doi: https://doi.org/10.1590/ S0047-20852012000300002

4. Soares FC, Flório FM, Zanin L. Satisfação dos usuários de Centros de Atenção Psicossocial. Rev. Latino-Am. Enfermagem. 2019;27:e3223. doi: https://doi. org/10.1590/1518-8345.3037.3223

5. Onocko-Campos R, Furtado JP, Trapé TL, Emerich BF, Surjus LTLS. Indicadores para avaliação dos Centros de Atenção Psicossocial tipo III: resultados de um desenho participativo. Saúde Debate. 2017;41(spe):71-83. doi: http://dx.doi.org/10.1590/0103-11042017s07

6. Kantorski LP, Jardim VMR, Coimbra VCC, Hypólito AM, Guedes AC, Meirelles MC, et al. Avaliando a política de saúde mental num CAPS: a trajetória no movimento antimanicomial. Estudos Pesqui Psicol. [Internet]. 2010;10(1);0-0. [Acesso 15 fev 2020] Disponível em: http://pepsic.bvsalud.org/scielo.php?script =sci_ arttext\&pid=S1808-42812010000100016\&lng=pt\&nrm $=$ iso

7. Trevisan E, Haas VJ, Castro SS. Satisfação e sobrecarga do trabalho nos Centros de Atenção Psicossocial Álcool e Drogas da Região do Triângulo Mineiro. Rev Bras Med Trab. 2019;17(4):511-20. doi: http://dx.doi.org/10.5327/ Z1679443520190434

8. Arantes IS, Sousa IF, Almeida RJ. Avaliação da satisfação profissional de trabalhadores em saúde mental. Espaço Saúde. 2016;17(1):93-101. doi: http://dx.doi. org/10.22421/1517-7130.2016v17n1p93

9. Lapischies SRC, Jardim VMR, Kantorski LP. Fatores associados à satisfação no trabalho em Centros de Atenção Psicossocial. Rev. Latino-Am. Enfermagem. 2014;22(6):950-8. doi: http://dx.doi. org/10.1590/0104-1169.3474.2500

10. Oliveira JF, Santos AM, Primo LS, Silva MRS, Domingues ES, Moreira FP, et al. Satisfação profissional e sobrecarga de trabalho de enfermeiros da área de saúde mental. Ciênc Saúde Coletiva. 2019;24(7):2593-9. doi: https://doi.org/10.1590/1413-81232018247.20252017 11. Dias GC, Furegato ARF. Impacto do trabalho e satisfação da equipe multiprofissional de um hospital psiquiátrico. Rev Enferm UERJ. [Internet]. 2016;24(1). doi: https://doi.org/10.12957/reuerj.2016.8164

12. Soares MH, Farinasso ALC, Gonçalves CS, Machado FP, Mariano LKFR, Santos CD. Sobrecarga e satisfação dos familiares de pacientes com esquizofrenia. Cogitare Enferm. 2019;24 (e) 54729. doi: http://dx.doi. org/10.5380/ce.v24i0.54729

13. Barbosa GC, Buesso TS. O impacto da sobrecarga de trabalho e a satisfação do trabalhador em saúde mental. Rev Saúde. (Santa Maria) 2019;45(2). doi: ttp://dx.doi. org/10.5902/2236583429678

14. Ishara S, Bandeira M, Zuardi AW. Public psychiatric services: job satisfaction evaluation. Rev Bras Psiquiatr. 2008;30(1):38-41. doi: http://dx.doi.org/10.1590/ S1516-44462006005000064

15. Ribeiro MC, Barros AC, Correia MS, Lessa RO, Tavares LN, Chaves JB. Atenção psicossocial e satisfação no trabalho: processos dialéticos na saúde mental. Rev Interdiscip. Estud. Saúde. [Internet]. 2018;7(1):55-67. [Acesso 22 abr 2020]. Disponível em: https://periodicos. uniarp.edu.br/index.php/ries/article/view/1102

16. Santos SS, Soares MH, Hirata AGP. Atitudes, conhecimento e opinião frente a saúde mental em alunos de graduação em enfermagem. Rev Esc Enferm USP. [Internet]. 2013;47(5);1195-202. doi: https://doi. org/10.1590/S0080-623420130000500026

17. Soares MH, Ruzzon ED, Bortoletto MSS. Concepção de profissionais de saúde que atuam emergência de saúde mental. SMAD, Rev Eletron Saúde Mental Álcool e Drogas. 2014;10(2):85-92. doi: http://dx.doi.org/10.11606/ issn.1806-6976.v10i2p85-92

18. Gonçalves AM, Vilela SC, Terra FS, Nogueira DA. Atitudes e o prazer/sofrimento no trabalho em saúde mental. Rev Bras Enferm. 2016;69(2):266-74. doi: http://dx.doi.org/10.1590/0034-7167.2016690209i

19. Mendes AOS, Pereira CM, Scattolin FAA. Opiniões de estudantes de um curso de graduação em Enfermagem sobre a doença mental. Rev Fac Ciênc Méd Sorocaba. 2019;21(2):69-76. doi: https://doi. org/10.23925/1984-4840.2019v21i2a6

\section{Contribuição dos autores}

Concepção e planejamento do estudo: Fernanda Pâmela Machado, Marcos Hirata Soares. Obtenção dos dados: Fernanda Pâmela Machado, Layla Karina Ferrari Ramos, Eloisi Delalibera Russon. Análise e interpretação dos dados: Fernanda Pâmela Machado, Marcos Hirata Soares. Análise estatística: Marcos Hirata Soares. Obtenção de financiamento: Fernanda Pâmela Machado, Marcos Hirata Soares, Layla Karina Ferrari Ramos, Eloisi 
Delalibera Russon. Redação do manuscrito: Fernanda Pâmela Machado, Layla Karina Ferrari Ramos, Eloisi Delalibera Russon. Revisão crítica do manuscrito: Fernanda Pâmela Machado, Marcos Hirata Soares.

Todos os autores aprovaram a versão final do texto. Conflito de interesse: os autores declararam que não há conflito de interesse.

Esta licença permite que outros distribuam, remixem, adaptem e criem a partir do seu trabalho, mesmo para fins comerciais, desde que the atribuam o devido crédito pela criação original. É a licença mais flexível de todas as licenças disponíveis. É recomendada para maximizar a disseminação e uso dos materiais licenciados. 